\title{
Paraplegia secundária à tuberculose multissistêmica: um relato de caso
}

\author{
Paraplegia secondary to multisystemic tuberculosis: a case report
}

Paraplegia secundaria a tuberculosis multisistémica: reporte de un caso

\begin{abstract}
Mariana Lima de Sousa ${ }^{1 *}$, Dênis Morgão Sertório², Gustavo Henrique Silva Almeida², Rafaella Ribeiro de Figueiredo², Rodrigo Tavares Silva², Wilson Cunha Júnior².
\end{abstract}

\section{RESUMO}

Objetivo: Aumentar a suspeição clínica para o diagnóstico de Tuberculose, auxiliar no reconhecimento e diagnóstico precoce dessa patologia e evitar desfechos de significativa morbidade e mortalidade. Detalhamento do caso: Paciente 41 anos, sexo masculino, com antecedente de etilismo de destilados e com passagens prévias em consultas nas unidades de pronto-atendimentoao longo de 1 ano. Buscou novamente auxílio médico, no momento apresentava uma doença em estágio avançado, com achados de fratura em vértebras torácicas de T9 a T11 e quadro de BexigaNeurogênica, associada à Pielonefrite que evoluiu com Doença Renal Crônica e necessidade de Terapia Renal Substitutiva. Durante a internação, foi diagnosticado: Tuberculose Miliar, Mal de Potte Tuberculose Renal, confirmados através de biópsia. Considerações finais: A tuberculose extrapulmonar deve ser investigada em pacientes provenientes de áreas endêmicas, quando os sintomas sugerirem quadro comuns a outras patologias se apresentando de forma crônica e com achados sistêmicos inespecíficos. Dessa forma, eleva-se a chance de cura.

Palavras-chave: Tuberculose extrapulmonar, Mal de Pott, Tuberculose renal, Paraplegia.

\begin{abstract}
Objective: To increase clinical suspicion for the diagnosis of Tuberculosis, assist in the recognitionand early diagnosis of this pathology and avoid significant morbidity and mortality outcomes. Case details: A 41-yearold male patient with a history of alcohol consumption of spirits and previous visits to emergency care units over the course of 1 year. He sought medical help again, at the time he had an advanced-stage disease, with findings of fracture in thoracic vertebrae from T9 to T11 and a picture of Neurogenic Bladder, associated with Pyelonephritis that evolved with Chronic Kidney Disease and the need for Renal Replacement Therapy. During hospitalization, it was diagnosed: Miliary Tuberculosis, Pott's Disease and Renal Tuberculosis, confirmed by biopsy. Final considerations: Extrapulmonary tuberculosis should be investigated in patients from endemic areas, when symptoms suggest a picture common to other pathologies, presenting chronically and with nonspecific systemic findings. In this way, the chance of cure is increased.
\end{abstract}

Keywords: Extrapulmonary tuberculosis, Pott's disease, Renal tuberculosis, Paraplegia.

\section{RESUMEN}

Objetivo: Incrementar la sospecha clínica para el diagnóstico de Tuberculosis, ayudar en el reconocimiento y diagnóstico precoz de esta patología y evitar resultados importantes de morbilidad y mortalidad. Detalles del caso: Paciente masculino de 41 años con antecedente de consumo dealcohol en licores y visitas previas a

1 Universidade CEUMA (UNICEUMA), São Luís - MA. *E-mail: mari_lima4@hotmail.com

${ }^{2}$ Centro Universitário Municipal de Franca (UNI-FACEF), Franca - SP.

SUBMETIDO EM: 12/2021

ACEITO EM: 12/2021

PUBLICADO EM: 12/2021 
urgencias en el transcurso de 1 año. Buscó nuevamente ayudamédica, en el momento en que presentaba una enfermedad en estado avanzado, con hallazgos defractura en vértebras torácicas de T9 a T11 y un cuadro de Vejiga Neurogénica, asociado a Pielonefritis que evolucionó con Enfermedad Renal Crónica y la necesidad de Reemplazo Renal. Terapia. Durante la hospitalización se diagnosticó: Tuberculosis Miliar, Enfermedad de Pott y Tuberculosis Renal, confirmada por biopsia. Consideraciones finales: La tuberculosis extrapulmonar debe investigarse en pacientes de áreas endémicas, cuando los síntomas sugieran un cuadro común a otras patologías, presentándose de forma crónica y con hallazgos sistémicos inespecíficos. De esta forma, se incrementa la posibilidad de curación.

Palabras clave: Tuberculosis extrapulmonar, Enfermedad de Pott, Tuberculosis renal, Paraplejía.

\section{INTRODUÇÃO}

A Tuberculose (TB) é uma doença infectocontagiosa de evolução crônica causada por um bacilo do gênero das Micobactérias, dentre os possíveis patógenos, a subespécie Mycobacterium tuberculosis, um bacilo aeróbio estrito, é a bactéria de maior relevância clínica em função de sua frequência e por apresentar como principal reservatório o ser humano (MINISTÉRIO DA SAÚDE, 2019).

Epidemiologicamente, essa doença permanece um problema de saúde pública global. Em 2019, a Organização Mundial de Saúde (OMS) estimava que um terço da população mundial estivesse infectada pelo bacilo. Destes, aproximadamente 10 milhões de pessoas teriam desenvolvido tuberculose, o que ocasionou cerca de 1,2 milhão de óbitos (MINISTÉRIO DA SAÚDE, 2021).

No Brasil, nesse mesmo ano, boletins do Sistema de Informação de Agravos de Notificação (SINAN) apontam o adoecimento de 37,4 indivíduos a cada 100 mil habitantes. Segundo dados do Sistema de Informações de Mortalidade, 4.532 evoluíram a óbito (MINISTÉRIO DA SAÚDE, 2021). Revelando que a tuberculose encontrou ambiente favorável frente à pobreza e desigualdade social do Brasil (MINISTÉRIO DA SAÚDE, 2021).

Sua transmissão ocorre pelo contato com aerossóis contendo o bacilo, advindos principalmente de pacientes com TB pulmonar e laríngea. Devido à maior frequência do acometimento pulmonar e por ser caracterizada como principal forma bacilífera em adultos, essa apresentação exibe maior relevância para a Saúde Pública e é considerada a principal responsável pela manutenção da transmissão (WORLD HEALTH ORGANIZATION (WHO), 2020).

Classicamente, as principais categorias do acometimento pulmonar são: TB pulmonar primária, TB pulmonar pós-primária e TB miliar. A tuberculose primária está relacionada ao primeiro contato em indivíduos não expostos previamente ao bacilo da tuberculose. Desse processo resulta uma reação inflamatória que pode determinar, desde achados de pneumonia extensa, até à disseminação da doença para linfonodos mediastinais e hilares ipsilaterais, essa linfonodopatia é visualizada em quase todos os pacientes (JULIO MEC, et al., 2021).

Trata-se de uma patologia comumente atribuída à infância, porém sua incidência tem aumentado entre adultos por todo o mundo. Os bacilos após inalação tendem a se depositar em terços médios e bases dos pulmões, supostamente pela maior ventilação pulmonar nessas áreas. No indivíduo imunocompetente há tendência à regressão do processo inflamatório. Quando a doençase perpetua após esse quadro inicial podese denominar Tuberculose Primária Progressiva, é encontrada em pacientes com comprometimento imunológico e caracterizada por apresentar achados de cavitações (BARBERIS I, et al., 2017).

Na Tuberculose pós-primária é essencial que o indivíduo seja previamente sensibilizado. Ocorre sejapor reinfecção ou reativação de bacilos inertes. Nessa forma da doença, porções apicaisde ambos os pulmões representam áreas de maior comprometimento, provavelmente decorrente da presença de mais bacilos viáveis nessas localidades em função da alta concentração de oxigênio (LEMOS CX, 2021). 
Diferente da TB primária, nesta há propensão à progressão do processo inflamatório com evolução para necrose caseosa, escavações e quando coalescentes com bronquíolos ou pequenos vasos sanguíneos,serão responsáveis, respectivamente, pela disseminação endobrônquica e hematogênica (SALDIVA PHN, et al., et al, 2006).

A forma miliar da TB pode ser secundária à apresentação primária ou pós-primária. É marcada pela liberação de êmbolos contendo o bacilo que podem alcançar o leito capilar de diversos órgãos após a disseminação hematogênica ou linfática, sendo o acometimento pulmonar, hepático e medular, respectivamente, os mais descritos (MERCÊS TMD, et al., 2021). Todos os órgãos e/ou sistemas estão suscetíveis à tuberculose, o que determina o surgimento de formas extrapulmonares. Quando os bacilos aspirados se proliferam e atingem os linfonodos, permanecem em um ciclo vicioso até alcançarem a corrente sanguínea (MINISTÉRIO DA SAÚDE, 2021).

A partir deste ponto, as bactérias estão livres para se disseminar por outros tecidos e, a depender doórgão acometido, irá provocar sintomas comuns a outras patologias e achados inespecíficos, através da presença de sintomas constitucionais sistêmicos. O acometimento extrapulmonar está descrito em até $15 \%$ dos casos (DHARMAPALAN A, et al., 2013).

Portanto, trata-se de uma doença grave, suspeitar de formas de Tuberculose Extrapulmonar e realizaro diagnóstico o mais precoce possível, bem como instituir o tratamento estão associados à maior probabilidade de cura e garantia de qualidade de vida para o doente (WHO, 2020). Com base no acima evidenciado, aumentar a suspeita clínica para o diagnóstico apresentado, ao servir de auxílio no reconhecimento e diagnóstico precoce dessa patologia, tem por propósito evitar desfechos de significativa morbidade e mortalidade.

\section{DETALHAMENTO DO CASO}

Paciente KST, masculino, 41 anos, pardo, lavrador e com escolaridade até $01^{\circ}$ grau incompleto. Apresentava antecedente de etilismo com consumo diário de destilados desde os 15 anos. Foi encaminhado do município onde residia via Central de Regulação de Ofertas de Serviços de Saúde Apresentava uma história complexa de queixa de dor lombar à esquerda, iniciada há um ano, com progressão para dorso à direita e consequente irradiação para membros inferiores; na ocasião, com diagnóstico de Infecção de Trato Urinário. Foi tratado com Ciprofloxacino, sem melhora do quadro clínico.

No período de um mês evoluiu com formigamento em ambos os membros inferiores e redução da força muscular progressiva, com dificuldade para deambular e desde então permaneceu acamado. Apresentava ainda relato de retenção urinária com necessidade de sondagem vesical de alívio intermitente, espasmos em MMII dolorosos e involuntários, dor lombar e perda ponderal de aproximadamente $30 \mathrm{~kg}$ em um ano. Negava trauma raquimedular, febre, sudorese e calafrios.

Em janeiro de 2021, durante seguimento em Centro de Reabilitação, ainda sem diagnóstico definitivo e em meio a tentativas de investigação, foi solicitado Ressonância Magnética de coluna tóraco- lombar, a qual não foi possível devido aos espasmos musculares que interferem na qualidade da imagem. Nova tentativa foi realizada com o uso do Baclofeno, indicado para estados espásticos nas mielopatias de diversas etiologias. O paciente evoluiu com os efeitos adversos, com desorientação, sonolência e confusão mental, descontinuando o tratamento.

Ao exame físico chamava atenção um paciente emagrecido, com tórax assimétrico e presença devértebra torácica proeminente em dorso. Os membros inferiores eram atróficos, com paraplegia espástica e sensibilidade reduzida até o nível da linha inframamilar. Avaliação do abdome sem alterações à inspeção, palpação e ausculta, Giordano Positivo bilateralmente.

A Tomografia Computadorizada de coluna tóraco-lombar evidencia lesões líticas destrutivas de T9 a T11 comprometendo os corpos vertebrais e os fragmentos ósseos proeminentes para o canal vertebral e comprimindo o saco tecal nestes níveis. 
Figura 1 - Segmento de vértebra torácica à nível de T10 representando corpo vertebral com lesões ósseas lítica e compressão de saco tecal.

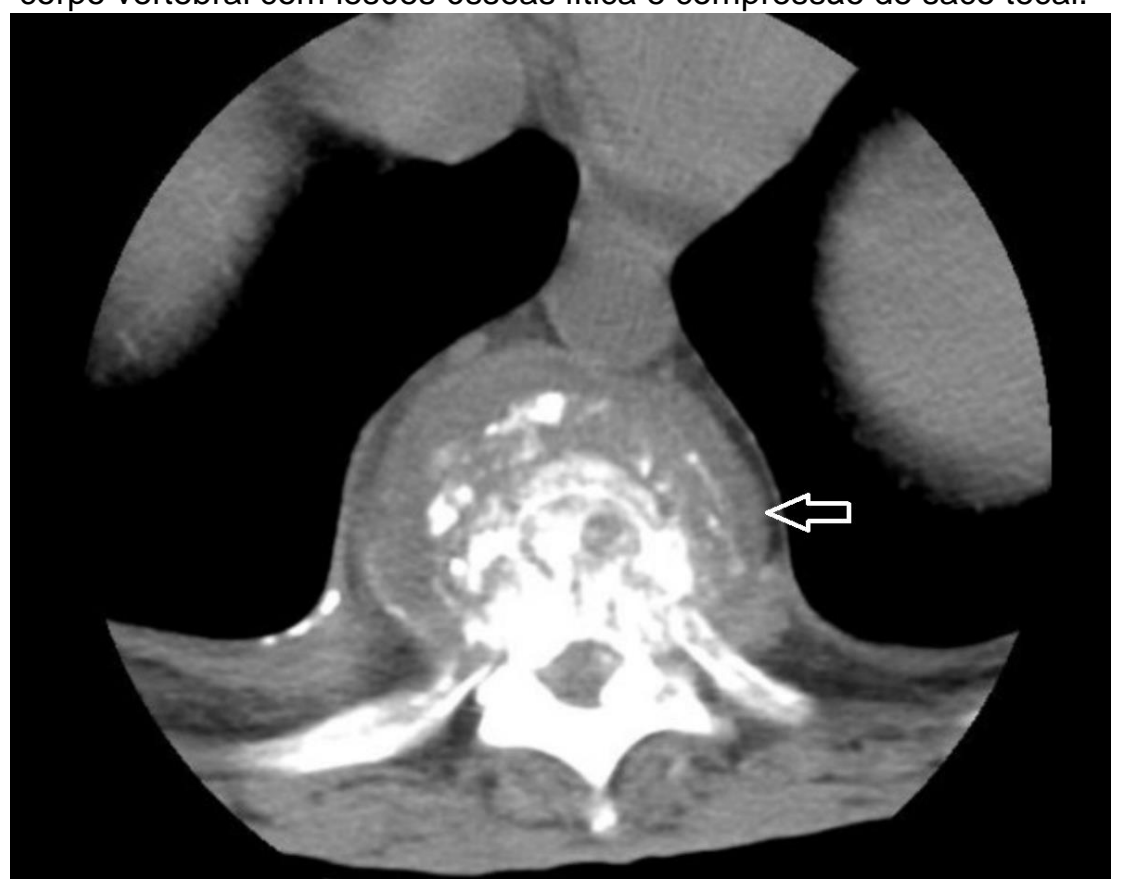

Fonte: Sousa ML, et al., 2021.

Alguns dias após a internação o paciente evoluiu com picos febris vespertinos e achado de crepitações por todo o tórax. Foi solicitado, então, uma tomografia de tórax para investigação, que trouxe sinais altamente sugestivos de tuberculose miliar.

A Tomografia Computadorizada do Tórax com achados de acometimento difuso dos múltiplos lobos pulmonares, marcado pela presença de nódulos que denotam sinais de disseminação broncogênica com distribuição randômica representada por nódulos em espaço aéreo e nódulos centrolobulares, configuram um padrão de árvore em brotamento (Figura 2).

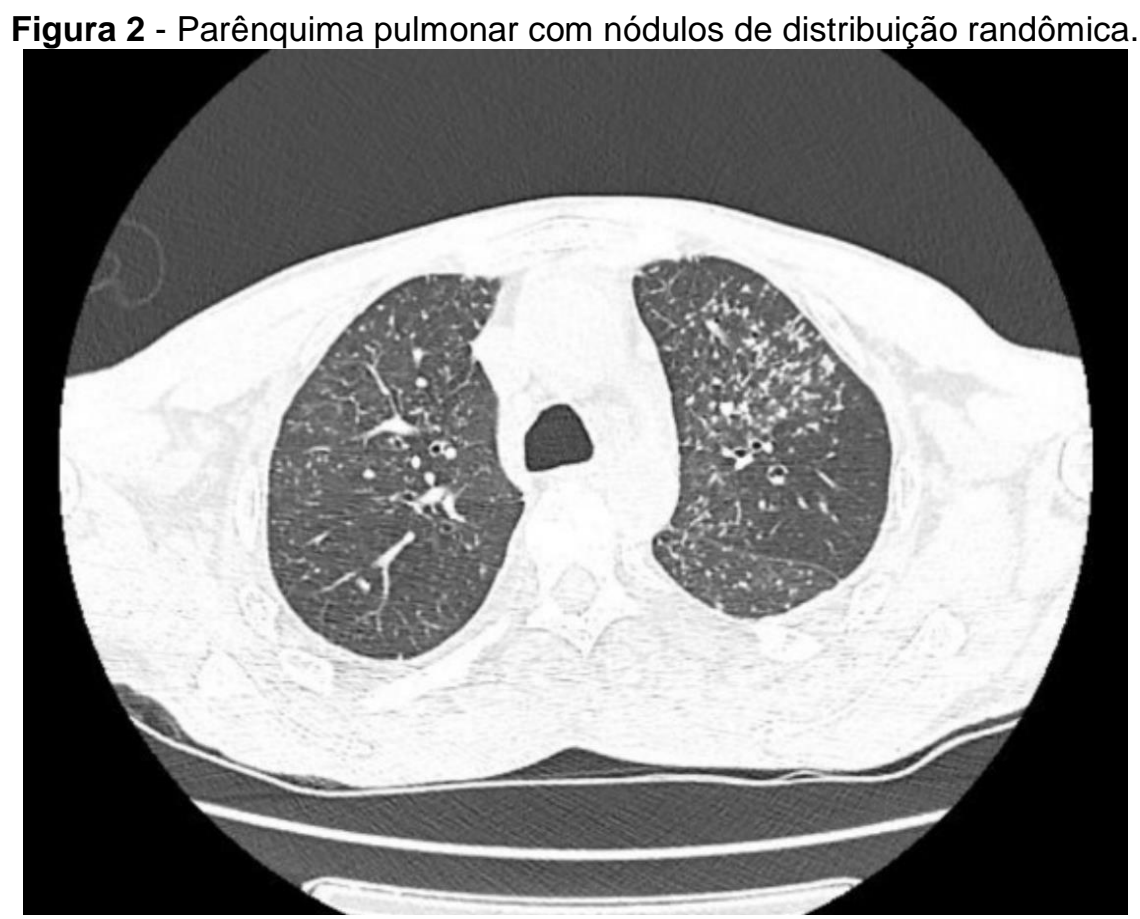

Fonte: Sousa ML, et al., 2021. 
Realizada biópsia de pulmão e pleura caseosa. Presença de infiltrado inflamatório com macrófagos e células gigantes multinucleadas, corado comhematoxilina e eosina e sem achado do bacilo, após coloração específica. Seguiu-se a investigaçãodiagnóstica com biópsia renal, uma vez que o paciente já estava em terapia renal substitutiva devido à doençarenal crônica.

Figura 3 - Imagem central determina a presença de necrose caseosa envolta por macrófagos e células gigantes multinucleadas.

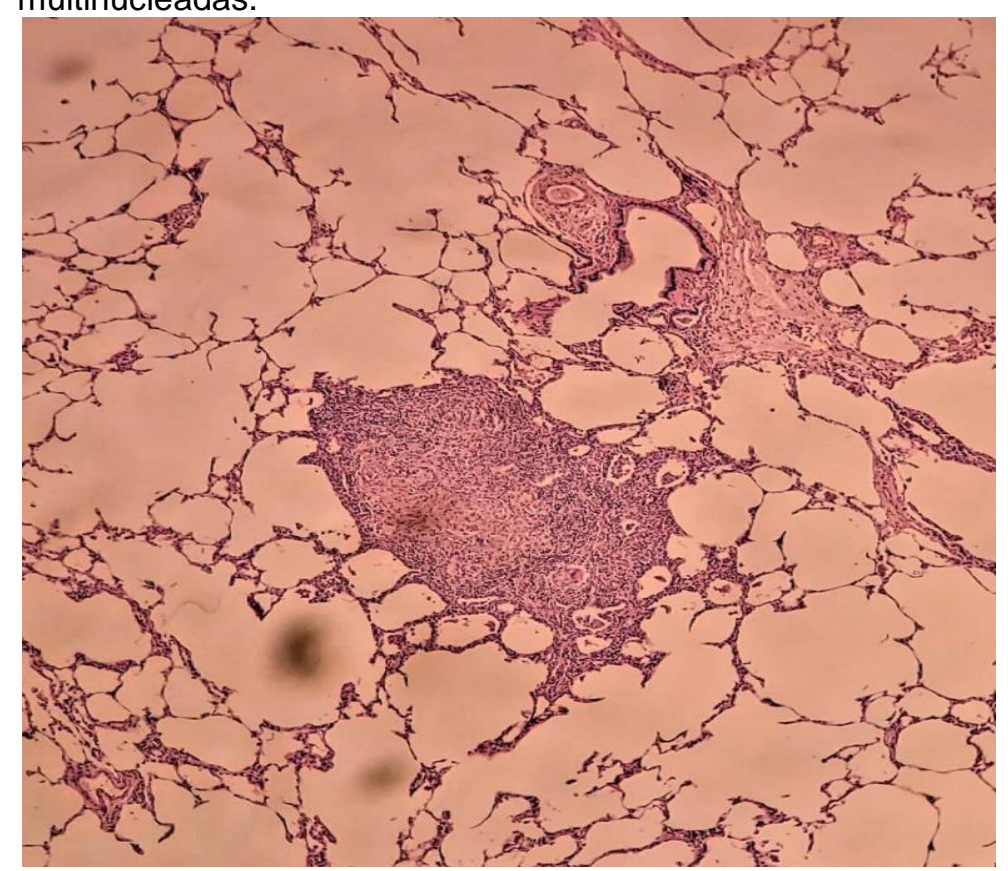

Fonte: Sousa ML, et al., 2021.

A Figura 4 representa o aparelho geniturinário com achados de padrão de hidronefrose associadoa pielonefrite crônica granulomatosa e necrose caseosa, apresentava linfonodo hilar com extensa necrose caseosa e ureter com processo inflamatório crônico granulomatoso, corado com hematoxilina e eosina, após coloração específica não são visualizados bacilos.

Figura 4 - Parênquima renal com infiltrado inflamatório com evidência de granuloma caseoso central.

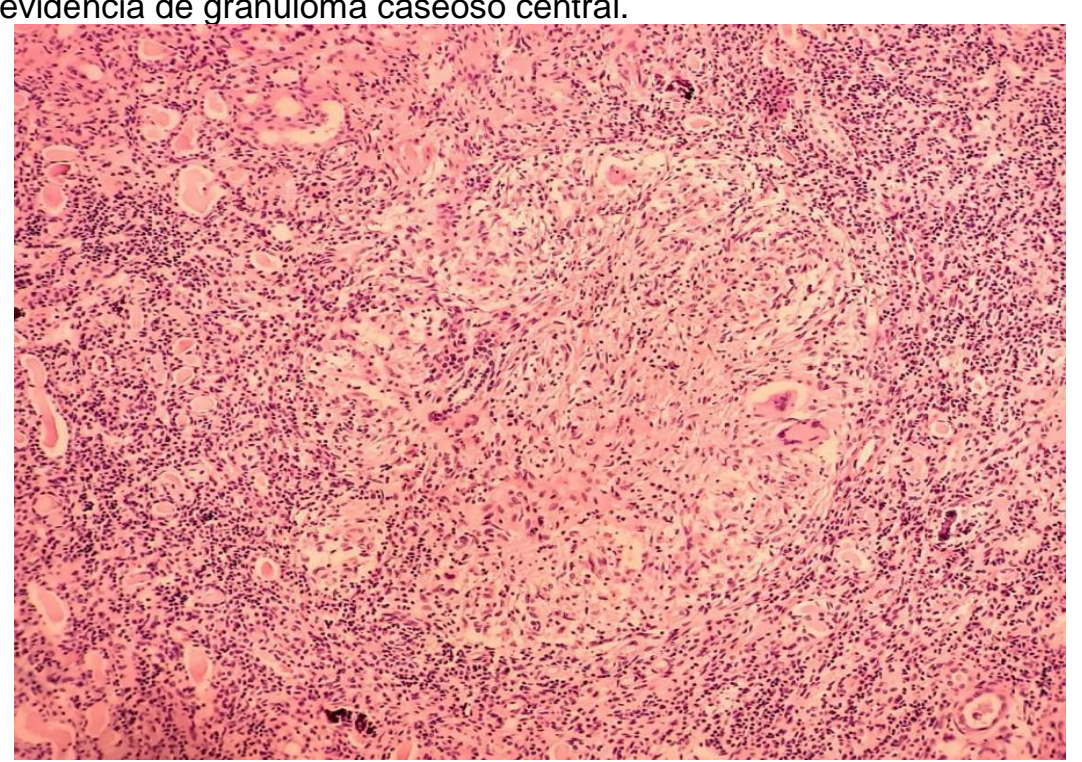

Fonte: Sousa ML, et al., 2021. 
Em busca de uma morbidade que justificasse a história clínica, os achados de exame físico, laboratório, imagem e histologia, fechou-se o diagnóstico de Tuberculose com acometimento multissistêmico.O paciente recebeu tratamento com Rifampicina, Isoniazida, Pirazinamida e Etambutol (RIPE) por dois meses, evoluindo com complicações dado o emagrecimento, internação prolongada e infecções nosocomiais de repetição, não resistindo ao tratamento proposto.

Dados coletados, informações descritas, exames de imagem, lâminas de material histopatológico e relato clínico foram autorizados pelo paciente com assinatura do Termo de Consentimento Livre Esclarecido (TCLE), descrito conforme orientações do Comitê de Ética em Pesquisa (CEP) e aprovado sob o número de Certificado de Apresentação de Apreciação Ética (CAAE) 49048521.2.0000.5438.

\section{DISCUSSÃo}

O Mycobacterium tuberculosis é um bacilo conhecido há aproximadamente 15.000 anos. Foi descritopor Robert Koch, por esse motivo, também é conhecido como Bacilo predominantemente inalatória, visto que há necessidade de oxigênio para o crescimento e multiplicação do patógeno, tornando o pulmão o primeiro órgão a ser atingido, uma vez que possui condições ideais para suaproliferação (MENEZES RF e MOURA ER, 2019).

Após 2 a 10 semanas do contato com a bactéria, a condição de infecção pode ser comprovada atravésdo Teste Tuberculínico (PPD). Entretanto, a probabilidade de a infecção tuberculosa evoluir com adoecimentogira em torno de $10 \%$ e está diretamente relacionada à carga bacteriana e ao sistema imunológico do indivíduo. Estatisticamente a TB pulmonar representa cerca de $70 \%$ dos casos relatados. Os outros sítios de infecção em ordem de frequência são linfonodos, pleura, trato urogenital, sistema musculoesquelético, trato intestinal, sistema nervoso central e doença disseminada (FALCETTA FS, et al., 2009).

Formas clínicas extrapulmonares se relacionam com a dificuldade do sistema imunológico de contera disseminação bacteriana. A Tuberculose Miliar, determinada pela implantação dos bacilos em diversos tecidos, com formação de lesões pequenas e arredondadas, apresenta prevalência de $5 \%$ e ocorre secundária à disseminação linfohematogênica. Trata-se de uma forma clínica de elevada mortalidade caso não seja diagnosticada e tratada precocemente. Clinicamente se apresenta com achados inespecíficos que podem não ser evidentes em Radiografia de Tórax. (SHARMA SK, et al., 2012).

A disseminação esquelética da tuberculose, conhecida como Mal de Pott, é caracterizada pelo acometimento de regiões torácicas inferiores e segmentos lombares da coluna vertebral. Apresenta caráter indolente com consequências devastadoras. A porção anterior do corpo vertebral é a parte anatômica em que se inicia a doença, a qual pode evoluir com inflamação, edema e necrose. Também é possível a formação de abscesso frio, com consequente compressão medular e desmielinização dos tratos espinhais. Os sintomas de maior severidade relatados são paraplegia, alteração esfincteriana associada à bexiga neurogênica e alterações sensitivas com dermátomos delimitados pelo nível da lesão (SILVA RD, et al., 2021).

Em relação ao acometimento renal, sabe-se que é secundário à disseminação hematogênica. Os bacilos atingem os rins pela porção cortical e determinam achados de granulomas que se concentram na região justaglomerular, com disseminação para o sistema urinário, provocando sintomas de polaciúria, noctúria, urgência miccional e disúria. Casos avançados desenvolvem uropatia obstrutiva, perda da função renal e hipertensão refratária (COSTA ACR, et al., 2020).

É válido ressaltar que a presença dos achados de leucocitúria e hematúria em indivíduos com urocultura negativa e nos quais após uso de antibioticoterapia incorre-se em falha de tratamento, prioritariamente em regiões endêmicas para a Tuberculose, deve suscitar a suspeita clínica com forte indicativo para a doença (JUNIOR GBS, et al., 2016)

O atraso no diagnóstico da Tuberculose Extrapulmonar leva a um desfecho com elevada morbidadee mortalidade. Defende-se que a pesquisa do bacilo em fragmentos microbiológicos deve ser realizada, 
apresentando como empecilho a dificuldade de obtenção das amostras de tecido por biópsia. Dados de literatura demonstram que em até 30 a $50 \%$ dos casos de tuberculose extrapulmonar se apresentam com baciloscopia negativa (MESQUITA CR, et al., 2010).

Dessa forma, o estudo histopatológico dos tecidos é considerado típico para a doença quando há presença de granulomas necrotizantes contendo macrófagos, linfócitos e células de Langhans. E, disposto em sua parte central, um conteúdo de degeneração progressiva denominado necrose caseosa (RAMÍREZ-LAPAUSA M, et al., 2015).

No Brasil o tratamento da Tuberculose é fornecido gratuitamente pelo SUS e seu recebimento se dá perante apresentação da ficha de notificação que deve ser preenchida pelo profissional da saúde que realiza o diagnóstico, uma vez que se trata de patologia com necessidade de notificação compulsória pelo Sistema de Informação de Agravos de Notificação (GADELHA PE, et al., 2014).

Desde 2009 o esquema para tratamento da Tuberculose no Brasil é composto por comprimido de dose combinada fixa compostos por $150 \mathrm{mg}$ de Rifampicina, $75 \mathrm{mg}$ de Isoniazida, $400 \mathrm{mg}$ de Pirazinamida e 275 mg de Etambutol, sendo este último o mais recente a ser introduzido ao esquema terapêutico padronizado. As doses, seguindo as devidas proporções, são ajustadas conforme o peso do paciente (RABAHI MF, et al., 2017).

Dessa forma, o esquema de tratamento antituberculose apresenta formulação via oral e se divide emduas fases. Na Tuberculose Pulmonar e nas formas extrapulmonares, exceto meningoencefalite, a fase intensiva é composta por 2 meses de tratamento com Rifampicina, Isoniazida, Pirazinamida e Etambutol. Seguido pela fase de manutenção com Rifampicina e Isoniazida durante 4 meses (MESQUITA CR, et al., 2021).

Caso haja interrupção do tratamento na fase intensiva por mais de 14 dias, será necessário recomeçaro esquema. Na fase de manutenção o ideal é que se completem as 120 doses no período de 7 meses, se 0 lapso acumulado for maior que 3 meses e as doses não atingirem $80 \%$ do tratamento, deve-se recomeçar o tratamento voltando à fase intensiva. Caso atingido mais que $80 \%$, a decisão será guiada por um novo Bacilo Álcool-ácido Resistente (BAAR) (RABAHI MF, et al., 2017).

Quando realizado o diagnóstico de Meningite secundária a presença do M. tuberculosis, o manejo do tratamento seguirá com 2 meses de terapia quádrupla na fase intensiva, seguida por 7 meses de fase de manutenção com terapia com Rifampicina e Isoniazida. Deve-se associar corticosteróide oral, com indicação de uso da prednisona de 1-2 mg/kg/dia por 4 semanas (IMRAN D, et al., 2020).

Pelo exposto, é notória a prevalência de formas da doença Tuberculose na população brasileira ao longo dos anos e sua incidência ainda incontestável nos dias atuais. Reconhecer sinais e sintomas e, quando côngruo, deve-se suscitar essa hipótese diagnóstica, no escopo de realizar o diagnóstico em fase inicial da doença, permitindo que sejam limitadas as injúrias pulmonares ou multissistêmicas. Definir a investigação, reconhecer a doença e fornecer o diagnóstico ao paciente, para abranger o acesso ao tratamento que é fornecido pelo SUS gratuitamente.

\section{REFERÊNCIAS}

1. BARBERIS I, et al. A história da Tuberculose: dos primeiros registros históricos ao isolamento do bacilo de Koch. Journal of preventive medicine and hygiene, 2017; 58(1): E9.

2. COSTA ACR, et al. Tuberculose Renal: relato de caso. Braz. J. Surg. Clin. Res. 2020; 30(3): 75-79.

3. DHARMAPALAN A, et al. Renal tuberculosis presenting as thigh abscess. The Indian Journal of Surgery, 2013. 75(1): 446-448.

4. FALCETTA FS, et al. Doença de Pott: uma doença milenar em um novo século. Revista HCPA 2009; $29: 2$.

5. GADELHA PE, et al. Controle da tuberculose: uma proposta de integração ensino-serviço. 7th ed. Rio de Janeiro: Fiocruz; 2014.

6. IMRAN D, et al. Estabelecendo a cascata de cuidados para pacientes com meningite tuberculosa. Wellcome Open Res 2020, 4: 177.

7. JULIO MEC, et al. Estudo comparativo dos critérios para o diagnóstico da tuberculose pulmonar infantil, Revista Residência Pediátrica, 2021; 11: 2-150 
8. JUNIOR GBS, et al. Doença Renal Crônica relacionada à Tuberculose Renal: um relato de caso. Revista Soc. Bras. Med. Trop. 2013; 49: 3.

9. KIM WS, et al. Tuberculose Pulmonar em crianças: avaliação com TC. Am J Roentgenol 1997; 168: $1005-1009$.

10. LEMOS CX. Manejo da tuberculose (TB) em unidade de tratamento intensivo: qual o impacto do uso de corticosteroides na TB pulmonar nesse contexto?, Dissertação (Mestrado em Ciências Pneumológicas), Universidade Federal do Rio Grande do Sul. 2021; 56p.

11. MENEZES RF, MOURA ER. PREVALÊNCIA DA TUBERCULOSE NO BRASIL NO PERÍODO DE 2011 A 2019 , 2021, 8(1): 534-545.

12. MERCÊS TMD, et al. Tuberculose miliar em pacientes com lúpus eritematoso sistêmico juvenil. Revista Multidisciplinar em Saúde, 2021, 2(4): 61.

13. MESQUITA CR, et al. Análise retrospectiva de casos de tuberculose em idosos. Revista Brasileira Em Promoção Da Saúde. 2021: 34.

14. MESQUITA M, et al. O diagnóstico tardio da Tuberculose Pulmonar levando à insuficiência renal irreversível em um paciente não imunocomprometido. Int Urol Nephrol. 2010. 42(1): 227-232.

15. MINISTÉRIO DA SAÚDE. Secretaria de Vigilância em Saúde. Brasil Livre da Tuberculose: Plano Nacional pelo Fim da Tuberculose como Problema de Saúde Pública, Brasil, 2017. Disponível em: https://bvsms.saude.gov.br/bvs/publicacoes/brasil_livre_tuberculose_plano_nacional.pdf. Acessado em: 06 de junho de 2021.

16. MINISTÉRIO DA SAÚDE. Secretaria de Vigilância em Saúde. Boletim Epidemiológico: Número Especial - Mar. 2021. Tuberculose 2021. Brasil, 2021. Disponível em: https://www.gov.br/saude/pt-br/media/pdf/2021/marco/24/boletimtuberculose-

2021_24.03\#: :text=Em\%202020\%2C\%200\%20Brasil\%20registrou,\%C3\%B3bitos\%20por\%20100\%20mil\%20habi tantes. Acessado em 06 de junho de 2021.

17. MINISTÉRIO DA SAÚDE. Departamento de Informática do SUS. Disponível em: https://antigo.saude.gov.br/images/pdf/2020/marco/24/Boletim-tuberculose-2020-marcas--1-.pdf.

18. MINISTÉRIO DA SAÚDE Doenças Transmissíveis. Manual de Recomendações para o Controle da Tuberculose no Brasil. Brasil, $2019 . \quad$ Disponível em: https://bvsms.saude.gov.br/bvs/publicacoes/manual_recomendacoes_controle_tuberculose_brasil_2_ed.pdf. Acessado em: 06 de junho de 2021. Acessado em: 06 de junho de 2021.

19. RABAHI MF, et al. Tratamento da Tuberculose, J Bras Pneumol. 2017; 43(5): 472-486

20. RAMIREZ-LAPAUSA M, et al. Tuberculose extrapulmonar, uma revisão de literatura. Rev Esp Sanid Penit. 2015; 17(1): 3-11.

21. SALDIVA PHN, et al. Pulmões. Pleura. In: Brasileiro G, editor. Bogliolo. Patologia. Rio de Janeiro: Guanabara Koogan, 2006: 358-407.

22. SHARMA SK, et al. Challenges in the diagnosis \& treatment of miliary tuberculosis. Indian J Med Res. 2012; 135(5): 703-30.

23. SILVA DR, et al. Consenso sobre o diagnóstico da tuberculose da Sociedade Brasileira de Pneumologia e Tisiologia. J. bras. Pneumol. 2021; 47(2)

24. WORLD HEALTH ORGANIZATION Global Tuberculosis Report 2020. Geneva, 2020. Disponível em: https://www.who.int/publications/i/item/97892400131311. 